\title{
The Dynamic Lives of Supermassive Black Holes in Merging Galaxies
}

\author{
Laura Blecha* \\ University of Maryland - College Park \\ E-mail: lblecha@astro.umd.edu
}

\begin{abstract}
Ample evidence suggests that the evolution of supermassive black holes and their host galaxies is connected, but the physical processes driving this link remain unclear. The role of galaxy mergers in triggering black hole growth and in creating the empirical black hole/galaxy bulge correlations is a matter of particularly active debate. Galaxy mergers are of additional interest as the precursors of supermassive black hole binaries, which may merge and produce powerful gravitational waves. This gravitational wave emission can also eject the merged black hole from its host nucleus, leaving the galaxy without a central black hole and producing an offset ("recoiling") quasar. There has been rapid recent progress from both theory and observations in constraining the merger/AGN connection and identifying signatures of active black hole pairs. I review these findings and discuss ongoing work to address remaining open questions. I also describe the handful of candidate recoiling black holes discovered to date, as well as results from numerical models that indicate promising avenues for identifying a population of such objects in current and future wide-field surveys.
\end{abstract}

BASH 2015

18 - 20 October, 2015

The University of Texas at Austin, USA

${ }^{*}$ Speaker. 


\section{Introduction and Motivation}

It is now well established that supermassive black hole (SMBH) masses are correlated with properties of their host galaxies, including stellar bulge mass, luminosity, and velocity dispersion [e.g., 1, 2, 3]. This suggests a physical link between SMBH growth and galaxy evolution on scales much larger than the SMBH sphere of influence. Mergers between galaxies are often invoked as a possible mechanism for driving coordinated growth of SMBHs and their hosts. Galaxy mergers increase the size of stellar bulges through dynamical heating, and they cause gravitational instabilities that can trigger new star formation and rapid fueling of the central SMBH [e.g., 4, 5, 6]. Feedback from star formation and active galactic nuclei (AGN) can in turn influence host galaxy evolution, for example by self-regulating nuclear activity and hastening the galaxy's transition to a "red and dead" elliptical [e.g., 7, 8, 9]

Galaxy mergers also inevitably lead to the formation of SMBH pairs, which, if both SMBHs are actively accreting, could be detected as dual AGN. These systems are of particular interest, as they provide unambiguous confirmation of an ongoing late-stage galaxy merger and point to highly favorable environments for rapid SMBH accretion. In addition, the SMBHs themselves can later merge, releasing enormous amounts of energy in gravitational waves (GWs). The field of GW astronomy was recently revolutionized by the first ever direct detection of GWs from the merger of two $\sim 30 \mathrm{M}_{\odot}$ BHs with LIGO [10], opening an entirely new window for exploring the Universe in GWs and providing a spectacular confirmation of general relativity in the strong-field regime. In the coming years, GWs from SMBH binary inspiral and merger could also be detected with pulsar timing arrays (PTAs) or a space-based interferometer such as eLISA [e.g., 11, 12].

In a generic merging SMBH system (with unequal masses and spins), the GW emission will be asymmetric. This imparts a "recoil kick" to the merged $\mathrm{BH}$, which can be large enough to eject the BH from the galaxy entirely [e.g., 13, 14]. If actively accreting, recoiling BHs could be detected as AGN that are spatially or kinematically offset from their hosts. These could provide the only evidence of SMBH mergers prior to direct GW detections.

The merger-driven paradigm is by no means a complete picture of SMBH and galaxy evolution. The importance of mergers in triggering AGN and setting the BH-bulge relations is still a matter of active debate, and attempts to quantify the connection between mergers and AGN empirically must overcome significant selection biases. Moreover, evidence for AGN pairs has been notoriously scarce even on large $(\mathrm{kpc})$ scales; until recently only a few serendipitously discovered pairs were known. Much progress has been made in recent years in identifying candidate kpc-scale dual AGN, opening a new window into the merger/AGN connection. The subsequent evolution of SMBH pairs on smaller scales is still somewhat uncertain, however, and while numerous SMBH binary candidates have been identified, none have yet been confirmed. The search for recoiling SMBHs is just beginning. Here I review recent progress in understanding merger-driven SMBH evolution, including ongoing efforts to identify signatures of SMBH inspiral, merger and recoil.

\section{When Do Mergers Matter?}

Numerous studies have demonstrated that the majority of AGN hosts do not shows signs of ongoing or recent merger activity, indicating that secular mechanisms are more common triggers of 
AGN fueling [e.g., 15, 16]. However, there is strong evidence that merger events do trigger AGN, in agreement with theoretical predictions. In samples of close galaxy pairs, the fraction that host at least one AGN increases with decreasing (projected) pair separation [17, 18, 19]. This is true for AGN selected at optical, hard X-ray, and near-IR wavelengths, and the dual AGN fraction shows an even stronger trend. These results strongly suggest a causal link between galaxy interactions and AGN fueling, where SMBHs are more likely to be active as the strength of the interaction increases.

Quantitatively, however, the empirical relationship between mergers and AGN is strongly influenced by selection effects. The above studies of AGN in galaxy pairs illustrate that the observed AGN fraction depends on merger stage and viewing angle. More importantly, the same mergerinduced instabilities that trigger gas inflow to the central SMBH can also trigger bursts of star formation. The copious amounts of dust produced by these starbursts can heavily obscure the nuclear region in ongoing mergers, weakening or masking entirely the signatures of AGN at optical and soft X-ray wavelengths. It has long been known that ultra-luminous infrared galaxies (ULIRGs), in which the bulk of emission is reprocessed by dust, commonly show signs of ongoing merger activity and contain obscured quasars [Figure 1; e.g., 20, 21]. In addition, a recent study of X-ray selected AGN found a correlation between the degree of AGN obscuration (column density) and the host merger fraction [22]. The most heavily obscured (Compton-thick) AGN have column densities $N_{\mathrm{H}}>10^{24} \mathrm{~cm}^{2}$ that attenuate even hard X-ray emission; studies indicate that Compton-thick AGN may comprise a significant fraction $(\sim 30-50 \%)$ of all AGN [e.g., 23, 24].

These empirical findings are well-supported by hydrodynamic simulations of galaxy mergers, which demonstrate that an obscured quasar phase should be generic to major, gas-rich mergers [e.g., 25, 26, 27]. Specifically, the peak obscuring column density and AGN luminosity should both occur during or shortly after the galaxies' final coalescence (Figure 2).

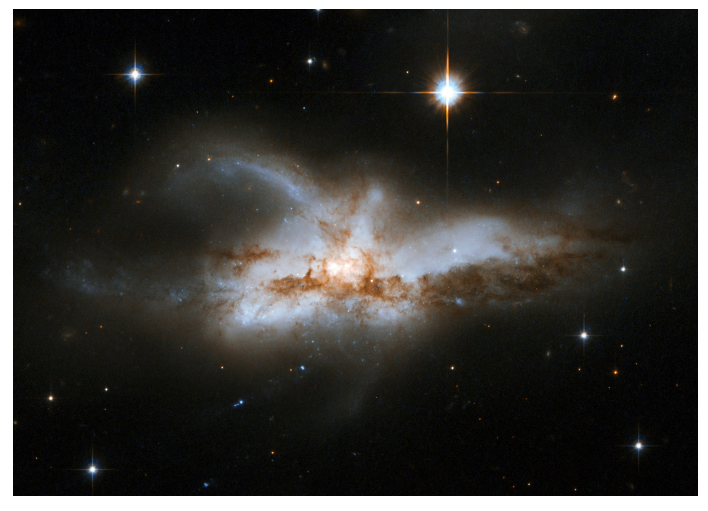

Figure 1: Hubble Space Telescope (HST) image of NGC 6240, a well-known ULIRG in the late stages of a major merger. Chandra observations have revealed two AGN in the nucleus that are separated by about a kpc; they are completely obscured at optical wavelengths [28]. Image credit: NASA, ESA, the Hubble Heritage Team (STScI/AURA)-ESA/Hubble Collaboration and A. Evans (University of Virginia, Charlottesville/NRAO/Stony Brook University).

The clear implication is that the fraction of merger-triggered AGN is likely to be underestimated in many surveys, because they are preferentially obscured. Indeed, when selected via near-IR signatures of AGN-heated dust (using data from the Wide-field Infrared Survey Explorer, WISE), AGN are found in a substantially higher fraction of merging galaxies than when optical selection is used [19]. A similar result is obtained for ultra-hard X-ray selected AGN; [29] found that 25\% of their Swift-BAT (14-195 keV) AGN sample are in apparent mergers, versus 4\% of AGN in a comparison sample selected via optical emission line diagnostics. 
The prediction that late-stage mergers often produce luminous quasars is supported by observations. Specifically, the fraction of AGN hosted in apparent mergers is a strong function of AGN luminosity. At the highest quasar luminosities (bolometric luminosity $L_{\mathrm{bol}} \gtrsim 10^{45-46} \mathrm{erg} \mathrm{s}^{-1}$ ), most AGN are hosted in major mergers [e.g., 30, 31]. Consequently, even though the majority of AGN by number are triggered by secular processes, merger-induced fueling is thought to dominate AGN population by luminosity and mass density [e.g., 32, 33, 34]. This suggests that mergers play a crucial role in setting the SMBH mass function and SMBH-bulge relations.
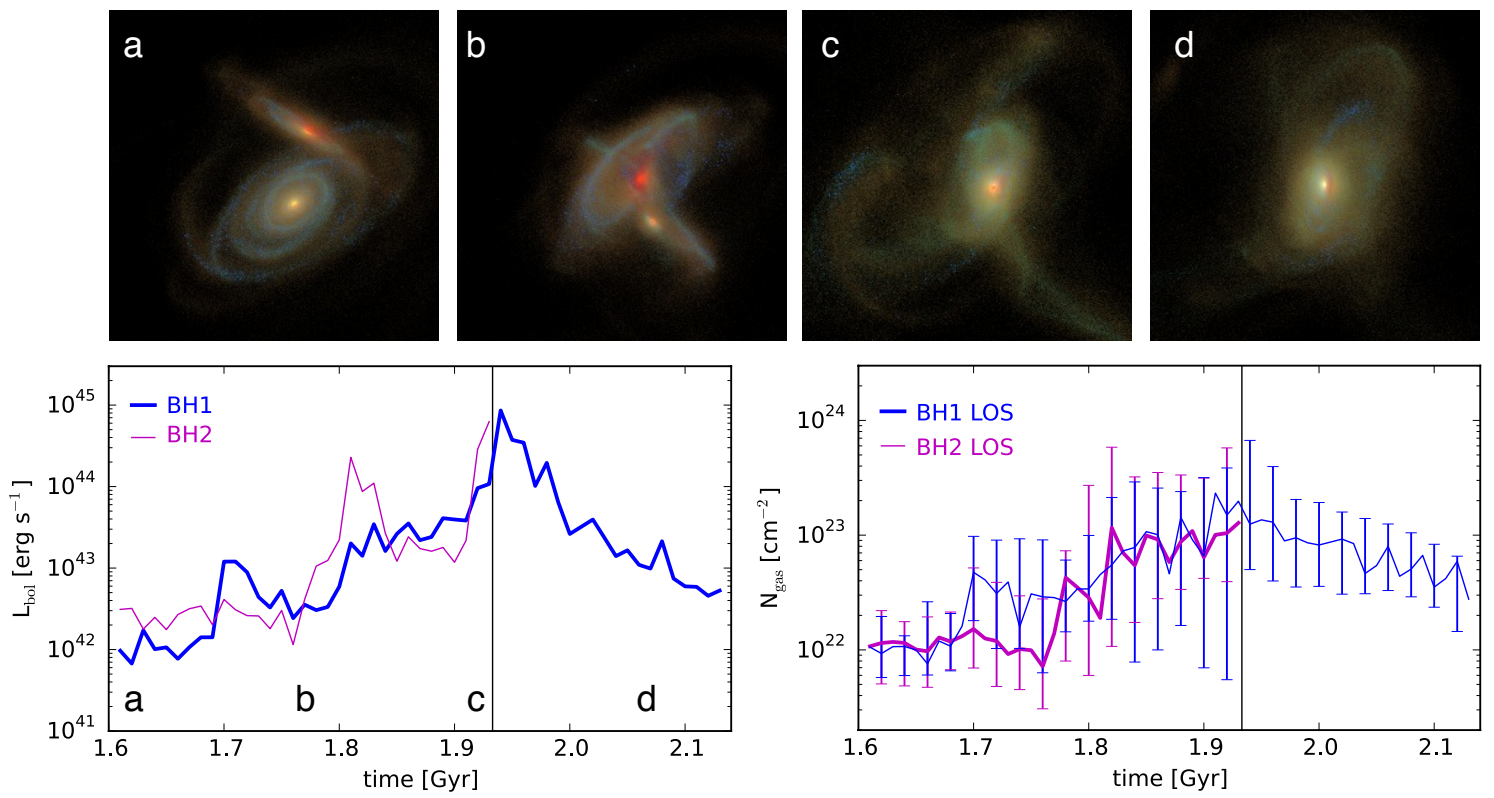

Figure 2: An illustrative simulated example of an obscured, luminous AGN pair triggered by a major merger. Top panels: Mock SDSS ugz snapshots of a gas-rich major galaxy merger (from SUNRISE radiative transfer applied to GADGET hydrodynamic simulation data). Bottom panels: For the same simulation, the evolution of AGN $L_{\text {bol }}$ and total gas column density $\left(\mathrm{N}_{\text {gas }}\right)$ along selected sight lines to each $\mathrm{BH}$. The error bars show the spread in $\mathrm{N}_{\text {gas }}$ over 7 viewing angles. The solid vertical line corresponds to the time of SMBH merger. The time of peak column density and peak AGN activity are clearly correlated, supporting the notion that obscured, luminous AGN are a natural occurrence in late-stage mergers.

\section{Dynamics and Observable Signatures of SMBH Pairs}

\subsection{Kiloparsec-scale Dual AGN}

Pairs of AGN can provide unique insight into merger-triggered fueling. As mentioned above, the fraction of galaxy pairs in which both galaxies host an AGN is a strong function of their physical separation, more so than for single AGN. These close AGN pairs are unambiguous tracers of latestage galaxy mergers, and the fact that both SMBHs are simultaneously active suggests that the nuclear environment in the merging system contains near-ideal conditions for rapid SMBH fueling.

The AGN pair in NGC 6240 [Figure 1; 28] was among the first to be identified on scales of $\lesssim 10 \mathrm{kpc}$, but further detections of close, spatially-resolved AGN pairs have been scarce [e.g., $35,36]$. As an alternate approach, several studies have focused on AGN with double-peaked narrow 
[OIII] emission line profiles [37, 38, 39]. This signature can be produced by the orbital motion of a kpc-scale SMBH pair, each with its own narrow line (NL) region. With these double-peaked NL AGN samples, the number of candidate dual AGN increased from a handful to several hundred.

However, double-peaked NL profiles can often be produced in single AGN systems, if for example the gas kinematics is dominated by outflowing winds or orbital motion in a clumpy or partially-obscured disk. Detailed follow-up is therefore required to confirm the presence of an AGN pair. In particular, systems in which two nuclei can be identified in both high-resolution imaging and resolved spectroscopy are strong dual AGN candidates (Figure 3). Follow-up studies indicate that at least $10-20 \%$ of double-peaked NL AGN are likely to be duals [e.g., 40, 41, 42]. In some cases, the dual AGN nature of these candidates has been confirmed by the detection of dual X-ray or flat-spectrum radio sources [e.g., 43, 44, 45].
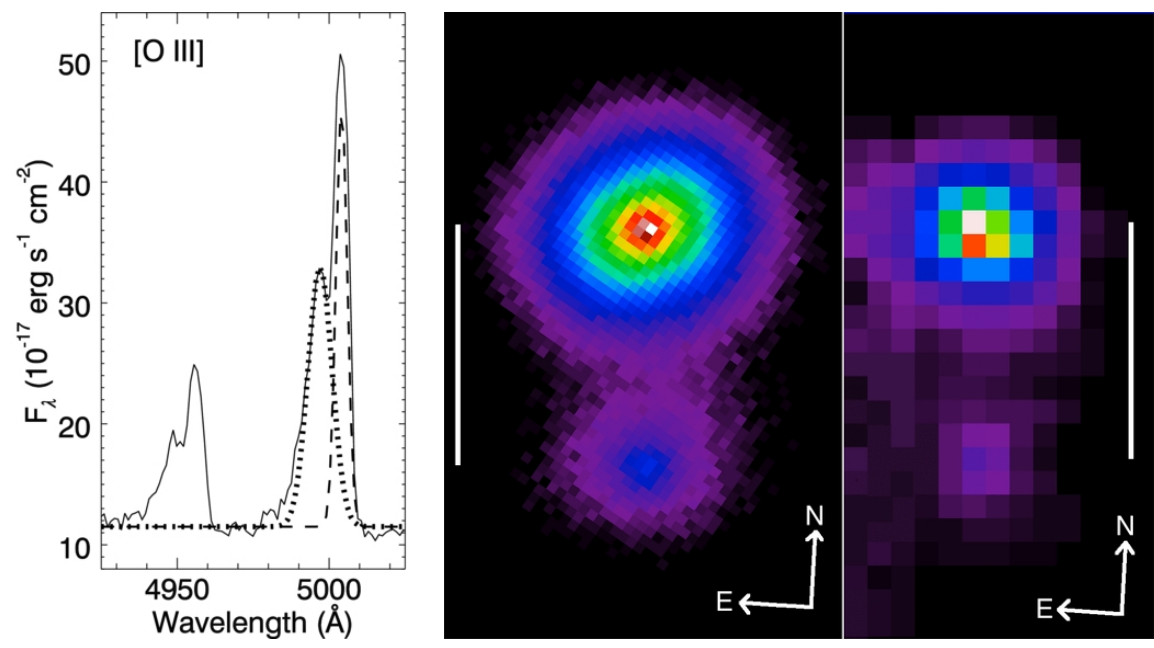

Figure 3: [From 46, Figs. 1 \& 3]. Example of an AGN pair (4.8 kpc separation) in SDSS J0952+2552, which was initially selected as a double-peaked NL AGN. Left: the [OIII] $\lambda 4959$ and [OIII] $\lambda 5008$ profiles, exhibiting clear double peaks. Middle \& right: Keck AO $H$-band imaging of the system with NIRC2 and OSIRIS, respectively (the OSIRIS image show the integrated flux in each spatial pixel). Both images reveal two spatially well-resolved components, corresponding to the dual AGN host galaxies.

The nature of many double-peaked NL AGN is still ambiguous, however. In order to better understand connection between double-peaked NL AGN and dual SMBHs, [47] developed a prescription for tracing NL gas kinematics in high-resolution hydrodynamic simulations of galaxy mergers. They find that double-peaked NL AGN are a generic but short-lived feature of gas-rich major mergers (Figure 4). Even within the kpc-scale phase of SMBH pair evolution, only a minority of double-peaked NL profiles are found to directly result from relative motion of the SMBHs. Most double-peaked NLs arise from gas kinematics in the dynamic merger environment. However, in many such cases the dual SMBH motion still influences NL profile. In [47] we identify some additional signatures, including large velocity separation or overall velocity shifts, that may point to the presence of an SMBH pair.

Biconical outflows are another important mechanism for producing double-peaked NL profiles around single AGN in isolated galaxies. Indeed, resolved spectroscopy of many double-peaked NL AGN reveals spatially extended emission, which likely signals the presence of outflowing winds 

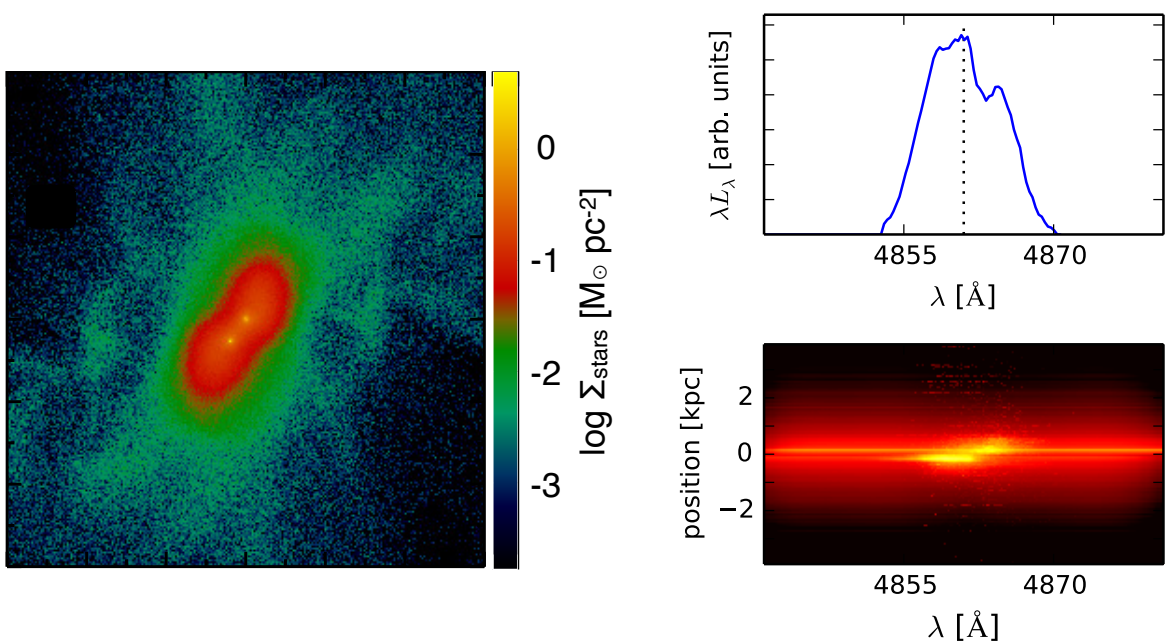

Figure 4: Left: From [47]. Example of a simulated AGN pair (2.3 kpc separation) in which orbital SMBH motion produces double-peaked NLs. The stellar density of the host galaxies is shown $20 \mathrm{Myr}$ prior to the SMBH merger, in a hydrodynamic galaxy merger simulation with the GADGET-3 code [48, 49]. Top right: double-peaked narrow $\mathrm{H} \beta$ profile produced by a kpc-scale AGN pair, generated with the SUNRISE dust radiative transfer code [50,51] from the simulations of [47]. The dotted line indicates the rest frame. Bottom right: same, but for a resolved "slit" spectrum. Two emission components well-separated in velocity and physical space are apparent, indicating that the double-peaked profile is produced by the dual SMBH.

[42, 52]. Roughly a third of these appear to be associated with kpc-scale radio jets [45]. These systems are interesting in their own right, as they present an opportunity to study the detailed kinematics and energetics of AGN feedback on sub-galactic scales. Moreover, although dual SMBHs account for only a minority of double-peaked NL AGN, these spectroscopically-selected samples have nonetheless greatly increased the number of known dual AGN.

\subsection{SMBH Binaries}

Below kpc scales, a SMBH pair inspirals via dynamical friction until it forms a gravitationallybound binary at separations of a few parsec. Spatially resolving such binaries in the nearby Universe is possible with very long baseline interferometry (VLBI). However, only one such system is known: the galaxy $0402+379$ has two compact radio cores separated by $7 \mathrm{pc}$ [53]. A subsequent search of $>3000$ objects with archival VLBI data yielded no further candidates, suggesting that binary radio-loud AGN are rare [54].

It is unclear whether the lack of observed binaries is primarily due to the difficulty in resolving them, or whether it indicates that they are short lived. The predicted timescales for SMBH binary inspiral depend strongly on environment. These range from a few Myr if the inspiral is driven by a cold, massive circumnuclear disk [e.g., 55, 56] to more than a Hubble time in gas-poor stellar cores, where the supply of gas and stars capable of driving inspiral may be depleted [the "final parsec problem"; e.g., 57, 58]. A cold gas reservoir is not required to evolve SMBH binaries beyond the parsec scale, however. If the stellar distribution is even slightly triaxial, low angular momentum stars will be continuously supplied to the galactic nucleus, where they can interact with the SMBH binary. And while simulating binary inspiral down to the GW-driven regime ( $\sim$ milliparsec scales) 
is still quite computationally challenging, recent work indicates that in most galactic nuclei, stellar dynamical processes should be capable of evolving SMBH binaries well past the "final parsec" in much less than a Hubble time [e.g., 59, 60, 61].

Spatially resolving sub-parsec binaries is essentially impossible with current instruments, but at $\sim$ milliparsec separations, periodic signatures in the light curve or SED that arise from the binary orbit could be observed on human timescales. No such binaries have yet been robustly confirmed, but a number of intriguing candidates have been identified [e.g., 62, 63, 64, 65, 66, 67]. Continued monitoring is needed to reveal which, if any, of these are binary SMBHs.

\section{Gravitational-Wave Recoil}

\subsection{Physical Mechanism and Predicted Signatures}

SMBH binary inspiral and merger events are extremely powerful GW sources. Pulsar timing arrays (PTAs) could detect GWs from individual massive $\left(>10^{9} \mathrm{M}_{\odot}\right)$ SMBH binaries at low redshift $(z \lesssim 1)$, and in the coming years they may detect the stochastic background of GWs from SMBH binaries [e.g., 68]. Current constraints on the GW background from PTAs are already beginning to place interesting limits on theoretical models of SMBH inspiral [e.g., 69]. In the future, a space-based interferometer such as eLISA could detect GWs from lower-mass SMBH merger events $\left(\sim 10^{5}-10^{6} \mathrm{M}_{\odot}\right)$ at redshifts as large as $\sim 20$ [e.g., 12].

Because merging SMBHs will generally have unequal masses and spins, the GWs are radiated asymmetrically. The resulting net linear momentum flux from the system imparts a "recoil kick" to the merged SMBH at the moment of merger [70,71]. Numerical relativity simulations indicate that these kicks may be up to $\sim 5000 \mathrm{~km} \mathrm{~s}^{-1}$ in some cases, far greater than galactic escape speeds $[14,72]$. While such extreme kicks should be exceedingly rare, even much smaller kicks would be astrophysically interesting. If the BHs are rapidly spinning $(a=0.9)$ and their spins are randomly oriented, $\sim 1 / 3$ of kicks will be $>500 \mathrm{~km} \mathrm{~s}^{-1}$. However, the kick velocity depends strongly on the SMBH spins just before merger, which are unknown. Theoretical work suggests that if the SMBHs are embedded in a circumnuclear gas disk, torques from the disk can align their spins with each other and with the disk angular momentum $[73,74,75]$. If the spins are perfectly aligned, the maximum recoil kick is $<200 \mathrm{~km} \mathrm{~s}^{-1}$. The efficiency of this process in real systems is not known; near-alignment of SMBH spins may be difficult to avoid if the binary inspiral is gas-driven, but little to no alignment may occur in cases where the inspiral is driven by stellar dynamics.

Gas dynamics can also have a large effect on the recoiling SMBH trajectory. In gas-rich galaxy mergers, cold gas inflow creates a steep potential well in the galactic nucleus during coalescence. This can efficiently prevent recoiling SMBHs from leaving the nuclear region, and gas dynamical friction further damps their orbital motion [76, 77, 78]. The outcome of a given recoil event therefore depends sensitively on the rapidly-varying galactic merger environment, in addition to the SMBH masses and spins.

If the SMBH has an accretion disk at the time of the kick, recoiling SMBHs could be detected as "AGN" that are spatially or kinematically offset from the actual galactic nucleus. These "offset AGN" may have substantial lifetimes, up to $\sim 100 \mathrm{Myr}$, for a wide range in kick speeds $[79,76]$. Velocity offsets could be detected as shifted broad emission lines (BLs) in the AGN SED; the BL 
region will remain bound to the recoiling $\mathrm{SMBH}$, while gas at larger radii (including the NL region) will be left behind. A confirmed detection of a recoiling AGN would provide evidence of a recent SMBH merger, the only such evidence that will be available without direct detection of GWs.

\subsection{Recoiling AGN Candidates}

Several candidate recoiling AGN have been identified in the last few years, though none have yet been confirmed [e.g., 80, 81, 82, 83, 84]. One of the most promising candidates, CID-42, is in a morphologically disturbed galaxy containing two optical nuclei separated by $2.5 \mathrm{kpc}$. These nuclei were initially proposed to be an inspiraling AGN pair [85]. However, a spectrum later revealed a $1300 \mathrm{~km} \mathrm{~s}^{-1}$ offset between the broad and narrow $\mathrm{H} \beta$ lines, suggesting that one of the nuclei instead corresponds to an recoiling AGN with a kinematic and spatial offset [83]. In this scenario, the other optical nucleus is simply the "empty" stellar cusp left behind. X-ray and radio data confirms that only one of these optical nuclei is producing AGN signatures [86, 87]. Theoretical modeling of this system also supports the recoil scenario, though it is difficult to rule out the presence of a quiescent SMBH in the secondary nucleus [88].

Another recoil candidate, SDSS 1133 , is a bright point source offset by $800 \mathrm{pc}$ from a nearby dwarf galaxy. The object is detected over a 65-year baseline and has an AGN-like spectrum with broad and narrow emission lines [84]. It is not yet possible to exclude an alternate scenario in which a long duration ( 50-year) luminous blue variable outburst was followed by an extremely long-lived Type IIn supernova, but in either case it is clear that SDSS 1133 is a highly unusual object without precedent. Further monitoring of this source should be able to distinguish between these scenarios in the near future.

\subsection{Toward Systematic Searches for Recoiling SMBHs}

The discoveries of recoiling AGN candidates to date have been mostly serendipitous; systematic searches have been hampered by large uncertainties. A few searches for velocity-offset quasars in SDSS spectra have been carried out, however [89, 63, 64]. [89] searched the DR5 quasar catalog for velocity shifts in multiple BLs and found a null result for $\Delta v>800 \mathrm{~km} \mathrm{~s}^{-1}$. [63] and [64] searched for offset broad $\mathrm{H} \beta$ lines in DR7 using slightly different selection criteria and found 32 and 88 velocity-offset quasars, respectively. Most of these are likely to be double-peaked emitters, high-velocity outflows, or a subparsec binary SMBH in which only one SMBH is active. Thus, it is clear that recoiling AGN with large velocity offsets are rare among SDSS quasars.

Spatially-offset AGN are largely unexplored thus far. Very small offsets $(<10 \mathrm{pc})$ have been found between the AGN and the host centroid in 6 of 14 core ellipticals, including M87 [82, 90]. However, the presence of a radio jet aligned with the displacement axis in four of these objects argues against GW recoil as an explanation. No searches have yet been carried out for recoiling AGN with larger spatial offsets.

In order to design targeted searches for offset AGN, better theoretical constraints on their observability are needed. [92] used semi-analytic merger tree models to predict the number of spatially-offset AGN that could be detected in surveys with HST, Chandra, and the James Webb Space Telescope (JWST). They predicted up to tens of offset AGN per square degree in the most optimistic case. However, as discussed above, gas dynamical effects can be very important in determining the outcome of recoil events. 

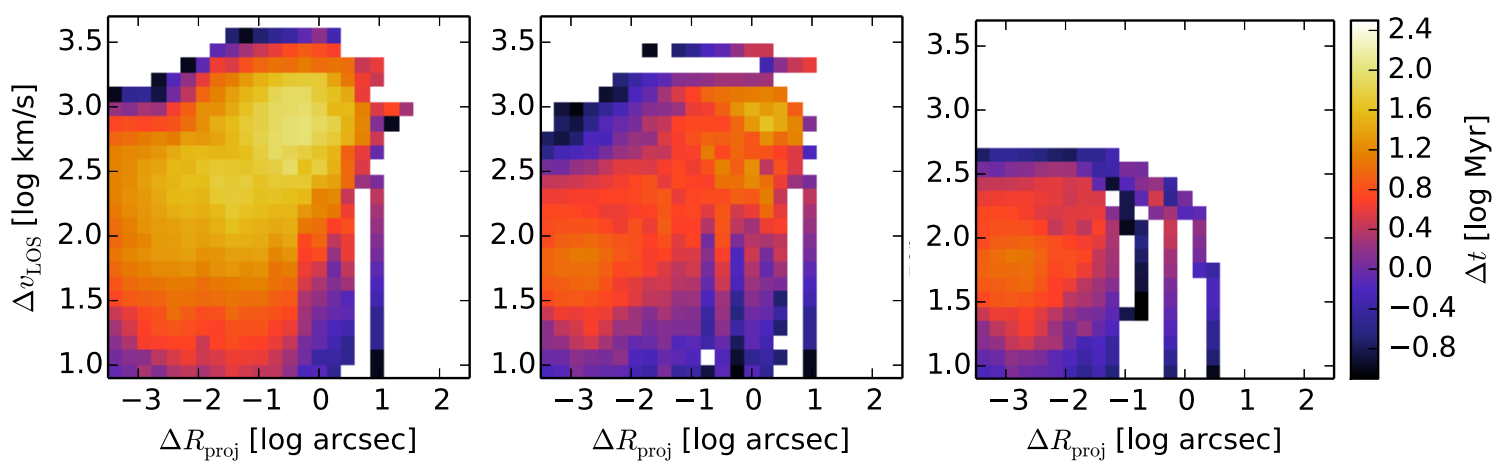

Figure 5: Time-weighted distributions of projected spatial offsets versus line-of-sight velocity offsets for recoiling AGN. The color scale indicates the amount of time a BH spends at a given $\left(\Delta R_{\text {proj }}, \Delta v_{\text {LOS }}\right)$ while actively fueled and offset from the host nucleus, integrated over all merger events, regardless of whether the event produces a resolvable offset. The sensitivity of HST-COSMOS is assumed. The left panel corresponds to a randomly-oriented spin model, the right panel assumes nearly-aligned spins, and the middle panel shows a hybrid model in which spin alignment occurs only in gas-rich mergers. Larger offsets are favored in the random and hybrid spin models but do not occur at all if BH spins are nearly aligned. From [91].

To this end, [91] have recently conducted a study of recoiling AGN that accounts for the effects of gas, using data from the Illustris cosmological hydrodynamic simulation [e.g., 93, 94, 95, 96]; this large-volume, high-resolution simulation was carried out with the moving-mesh hydrodynamics code AREPO [97]. After extracting SMBH merger events and host galaxy properties from the simulation, a model for recoiling AGN was implemented in post-processing, including BH spin alignment and recoil dynamics based on the gas-richness of host galaxies.

Figure 5 shows the time-weighted distributions of spatial and velocity offsets for recoiling AGN over cosmic time, based on the models of [91]. If SMBH spins are always aligned to within a few degrees, recoils are unlikely to be detected. In less extreme models of spin alignment, the distribution is dominated by large offsets, in part because kick velocities near the host escape speed yield the longest observable offset lifetimes. Crucially, this means that even seeing-limited observations could resolve spatially-offset AGN, making them promising targets for large-area surveys. In the most optimistic models, where spins are always randomly oriented, nearly a thousand velocityoffset AGN are predicted within the SDSS footprint. While this cannot be compared at face value with the rarity of large BL offsets observed among SDSS quasars, owing to selection effects, it provides the first tentative evidence that spin alignment plays a role in suppressing recoils.

Nonetheless, in the most physically-motivated models where spin alignment is efficient only in gas-rich mergers, hundreds of offset AGN are predicted to be observable in surveys with the Panoramic Survey Telescope \& Rapid Response System (Pan-STARRS), the Large Synoptic Survey Telescope (LSST), Euclid, and the Wide-Field Infrared Survey Telescope (WFIRST) (Figure 6). These findings strongly motivate a dedicated search for offset AGN.

\section{Summary and Future Outlook}

Disparate results from studies of the merger/AGN connection have generated a great deal of 

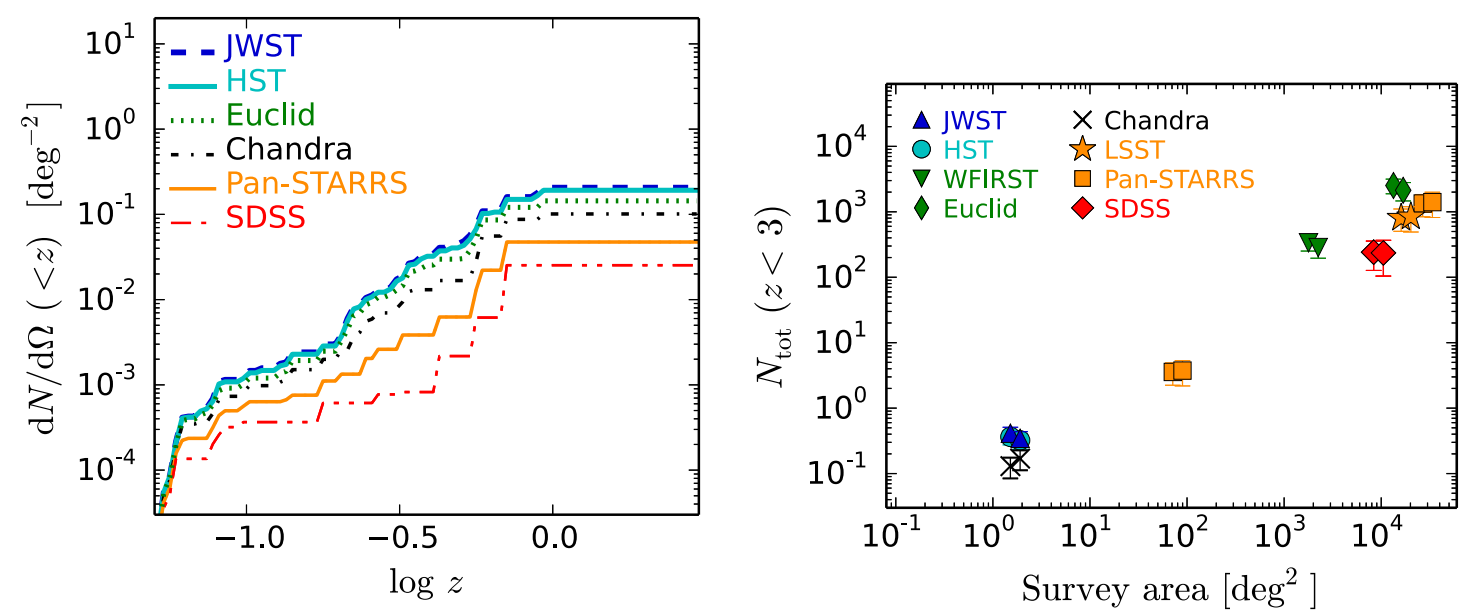

Figure 6: From [91]. Left: cumulative number of spatially-offset AGN observable per $\mathrm{deg}^{2}$ as a function of redshift. The model shown assumes that spins are nearly aligned in gas-rich mergers and randomly oriented in gas-poor mergers. Each curve assumes the sensitivity and resolution of a given instrument/survey as indicated on the plot legends. In each case, the minimum detectable offset $\Delta R_{\text {proj }}$ is assumed to be twice the resolution limit. Right: The total predicted number of observable spatially offset AGN at $z<3$ is shown for various surveys, as indicated in the plot legend. Survey sensitivity and resolution is the same as assumed in the left panel, though here we also show results for the WFIRST high-latitude survey and the LSST Main Survey. Large populations of recoiling AGN could be detected in all-sky surveys.

controversy about the role of galaxy mergers in $\mathrm{BH}$ and galaxy evolution. However, much of this uncertainty can be attributed to selection biases and sample incompleteness in AGN surveys. The tendencies for merger-triggered AGN to be preferentially obscured and highly luminous are particularly important, but other effects also play a role. These include surface brightness dimming of stellar tidal features, projection effects, stochasticity of AGN fueling, redshift evolution, and delays between galaxy interactions and accretion onto the SMBH.

With this in mind, a self-consistent picture has emerged in which SMBH growth is dominated by efficient, merger-induced quasar phases, while the large majority of AGN by number are low- to moderate-luminosity events triggered by secular processes. The quantitative details of this scenario are still uncertain, however. In particular, multiwavelength studies of AGN in galaxy pairs and mergers will be crucial for constraining the effects of obscuration. Larger statistical samples of single and dual AGN in mergers are also needed to account for other observational biases. $\check{\mathrm{N}}$

Numerous other open questions regarding SMBH/galaxy co-evolution remain. For one, the primary fueling modes of isolated AGN are not well understood. Minor mergers or close satellite encounters, gas infall onto host galaxies, or secular processes such as bar instabilities are all possible contributors, and the dominant mode likely varies with galaxy mass, redshift, and environment. Establishing a causal link to AGN fueling is nontrivial, however. For example, little to no correlation has been found between stellar bars and AGN [e.g., 98, 99]. Regardless of what triggers the initial gas inflow, a series of instabilities at different radii may ultimately be responsible for transporting gas to the smallest scales [e.g., 100, 101].

One of the greatest current challenges in understanding the SMBH-bulge relations is the difficulty in obtaining reliable BH masses. Dynamical mass measurements are the most robust but 
require resolving the SMBH sphere of influence. Reverberation mapping can provide mass measurements for active SMBHs and has the advantage that it can be used at $z>0$. Most SMBH mass estimates are obtained through more indirect means, however, and galaxy bulge properties are also increasingly difficult to measure at high $z$. Nonetheless, increasing evidence suggests that the SMBH-bulge relations evolve with redshift, with SMBH masses lying above the relation at high $z$ [e.g., 102, 103]. This is expected if indeed the bulk of SMBH mass is accreted in mergertriggered quasar phases, because merging and quasar activity peaks at $z \sim 2-3$. In the coming years, large reverberation mapping campaigns could greatly increase the number of SMBH mass measurements. Looking further ahead, thirty-meter-class telescopes would revolutionize our ability to obtain dynamical SMBH masses.

The role of galaxy mergers in producing GW sources - namely, SMBH binary mergers - is also of great current interest. Following the recent direct detection of GWs by LIGO, the future of GW astronomy appears extremely bright. In the SMBH regime, PTAs may make a detection of the stochastic GW background within the decade, and the LISA Pathfinder mission is currently paving the way for a space-based interferometer that could directly detect GWs from SMBH mergers. Moreover, the upcoming era of time-domain astronomy will greatly aid the search for close SMBH binaries, which would constrain GW event rates and the nature of the accretion flow prior to SMBH merger. This is also an ideal time to undertake dedicated searches for recoiling AGN, as large surveys could reveal a substantial population of such objects. Even a single confirmed recoiling SMBH would provide evidence of a recent SMBH merger and would constitute an independent test of strong-field gravity.

\section{Acknowledgements}

The author would like to thank the organizers for their kind invitation and hospitality.

\section{References}

[1] J. Magorrian, S. Tremaine, D. Richstone et al., The Demography of Massive Dark Objects in Galaxy Centers, AJ 115 (June, 1998) 2285-2305, [astro-ph/ 9708072 ].

[2] S. Tremaine, K. Gebhardt, R. Bender et al., The Slope of the Black Hole Mass versus Velocity Dispersion Correlation, ApJ 574 (Aug., 2002) 740-753, [astro-ph/ 02034 68].

[3] N. J. McConnell and C.-P. Ma, Revisiting the Scaling Relations of Black Hole Masses and Host Galaxy Properties, ApJ 764 (Feb., 2013) 184, [arXiv:1211.2816].

[4] D. B. Sanders, B. T. Soifer, J. H. Elias et al., Ultraluminous infrared galaxies and the origin of quasars, ApJ 325 (Feb., 1988) 74-91.

[5] J. E. Barnes and L. Hernquist, Transformations of Galaxies. II. Gasdynamics in Merging Disk Galaxies, ApJ 471 (Nov., 1996) 115-+.

[6] J. C. Mihos and L. Hernquist, Gasdynamics and Starbursts in Major Mergers, ApJ 464 (June, 1996) 641-+, [astro-ph/9512099]. 
[7] J. S. B. Wyithe and A. Loeb, Self-regulated Growth of Supermassive Black Holes in Galaxies as the Origin of the Optical and X-Ray Luminosity Functions of Quasars, ApJ 595 (Oct., 2003) 614-623, [astro-ph/0304156].

[8] T. Di Matteo, V. Springel, and L. Hernquist, Energy input from quasars regulates the growth and activity of black holes and their host galaxies, Nature $\mathbf{4 3 3}$ (Feb., 2005) 604-607, [astro-ph/0502199].

[9] P. F. Hopkins, T. J. Cox, D. Kereš et al., A Cosmological Framework for the Co-evolution of Quasars, Supermassive Black Holes, and Elliptical Galaxies. II. Formation of Red Ellipticals, ApJS 175 (Apr., 2008) 390-422, [arXiv: 0706.1246$].$

[10] B. P. Abbott, R. Abbott, T. D. Abbott et al., Observation of Gravitational Waves from a Binary Black Hole Merger, Physical Review Letters 116 (Feb., 2016) 061102, [arXiv:1602.03837].

[11] A. Sesana, A. Vecchio, and C. N. Colacino, The stochastic gravitational-wave background from massive black hole binary systems: implications for observations with Pulsar Timing Arrays, MNRAS 390 (Oct., 2008) 192-209, [arXiv : 0804 . 44 76].

[12] P. Amaro-Seoane, S. Aoudia, S. Babak et al., Low-frequency gravitational-wave science with eLISA/NGO, Classical and Quantum Gravity 29 (June, 2012) 124016, [arXiv:1202.0839].

[13] J. A. González, M. Hannam, U. Sperhake et al., Supermassive Recoil Velocities for Binary Black-Hole Mergers with Antialigned Spins, Physical Review Letters 98 (June, 2007) 231101-+, [gr-qc/0702].

[14] M. Campanelli, C. O. Lousto, Y. Zlochower et al., Maximum Gravitational Recoil, Physical Review Letters 98 (June, 2007) 231102-+, [gr-qc/ 0702 ].

[15] M. Cisternas, K. Jahnke, K. J. Inskip et al., The Bulk of the Black Hole Growth Since $z \sim 1$ Occurs in a Secular Universe: No Major Merger-AGN Connection, ApJ 726 (Jan., 2011) 57, [arXiv:1009.3265].

[16] D. D. Kocevski, S. M. Faber, M. Mozena et al., CANDELS: Constraining the AGN-Merger Connection with Host Morphologies at z 2, ApJ 744 (Jan., 2012) 148, [arXiv:1109.2588].

[17] S. L. Ellison, D. R. Patton, J. T. Mendel et al., Galaxy pairs in the Sloan Digital Sky Survey - IV. Interactions trigger active galactic nuclei, MNRAS 418 (Dec., 2011) 2043-2053, [arXiv:1108.2711].

[18] M. Koss, R. Mushotzky, E. Treister et al., Understanding Dual Active Galactic Nucleus Activation in the nearby Universe, ApJ 746 (Feb., 2012) L22, [arXiv:1201.2944].

[19] S. Satyapal, S. L. Ellison, W. McAlpine et al., Galaxy pairs in the Sloan Digital Sky Survey - IX. Merger-induced AGN activity as traced by the Wide-field Infrared Survey Explorer, MNRAS 441 (June, 2014) 1297-1304, [arXiv: 1403.7531$].$ 
[20] D. B. Sanders and I. F. Mirabel, Luminous Infrared Galaxies, ARA\&A 34 (1996) 749-+.

[21] S. Veilleux, D. S. N. Rupke, D.-C. Kim et al., Spitzer Quasar and Ulirg Evolution Study (QUEST). IV. Comparison of 1 Jy Ultraluminous Infrared Galaxies with Palomar-Green Quasars, ApJS 182 (June, 2009) 628-666, [arXiv: 0905 .1577].

[22] D. D. Kocevski, M. Brightman, K. Nandra et al., Are Compton-thick AGNs the Missing Link between Mergers and Black Hole Growth?, ApJ 814 (Dec., 2015) 104, [arXiv:1509.03629].

[23] Y. Ueda, M. Akiyama, K. Ohta et al., Cosmological Evolution of the Hard X-Ray Active Galactic Nucleus Luminosity Function and the Origin of the Hard X-Ray Background, ApJ 598 (Dec., 2003) 886-908, [astro-ph / 0308140 ].

[24] J. Buchner, A. Georgakakis, K. Nandra et al., Obscuration-dependent Evolution of Active Galactic Nuclei, ApJ 802 (Apr., 2015) 89, [arXiv: 1501.02805 ].

[25] P. F. Hopkins, L. Hernquist, T. J. Cox et al., A Unified, Merger-driven Model of the Origin of Starbursts, Quasars, the Cosmic X-Ray Background, Supermassive Black Holes, and Galaxy Spheroids, ApJS 163 (Mar., 2006) 1-49, [ast ro-ph/ 0506398 ].

[26] D. Narayanan, A. Dey, C. C. Hayward et al., A physical model for $z \sim 2$ dust-obscured galaxies, MNRAS 407 (Sept., 2010) 1701-1720, [arXiv: 0910.2234 ].

[27] G. F. Snyder, C. C. Hayward, A. Sajina et al., Modeling Mid-infrared Diagnostics of Obscured Quasars and Starbursts, ApJ 768 (May, 2013) 168, [arXiv:1210.6347].

[28] S. Komossa, V. Burwitz, G. Hasinger et al., Discovery of a Binary Active Galactic Nucleus in the Ultraluminous Infrared Galaxy NGC 6240 Using Chandra, ApJ 582 (Jan., 2003) L15-L19, [astro-ph/0212099].

[29] M. Koss, R. Mushotzky, S. Veilleux et al., Merging and Clustering of the Swift BAT AGN Sample, ApJ 716 (June, 2010) L125-L130, [arXiv: 1006 . 0228].

[30] T. Urrutia, M. Lacy, and R. H. Becker, Evidence for Quasar Activity Triggered by Galaxy Mergers in HST Observations of Dust-reddened Quasars, ApJ 674 (Feb., 2008) 80-96, [arXiv:0709.2805].

[31] E. Treister, K. Schawinski, C. M. Urry et al., Major Galaxy Mergers Only Trigger the Most Luminous Active Galactic Nuclei, ApJ 758 (Oct., 2012) L39, [arXiv:1209. 5393].

[32] A. C. Fabian, The obscured growth of massive black holes, MNRAS 308 (Oct., 1999) L39-L43, [astro-ph/9908064].

[33] A. R. Draper and D. R. Ballantyne, A Tale of Two Populations: The Contribution of Merger and Secular Processes to the Evolution of Active Galactic Nuclei, ApJ 751 (May, 2012) 72, [arXiv:1203.5117]. 
[34] P. F. Hopkins, D. D. Kocevski, and K. Bundy, Do we expect most AGN to live in discs?, MNRAS 445 (Nov., 2014) 823-834, [arXiv: 1309.6321$].$

[35] S. Bianchi, M. Chiaberge, E. Piconcelli et al., Chandra unveils a binary active galactic nucleus in Mrk 463, MNRAS 386 (May, 2008) 105-110, [arXiv: 0802 . 0825].

[36] P. J. Green, A. D. Myers, W. A. Barkhouse et al., SDSS J1254+0846: A Binary Quasar Caught in the Act of Merging, ApJ 710 (Feb., 2010) 1578-1588, [arXiv:1001.1738].

[37] J. M. Comerford, B. F. Gerke, J. A. Newman et al., Inspiralling Supermassive Black Holes: A New Signpost for Galaxy Mergers, ApJ 698 (June, 2009) 956-965, [arXiv:0810.3235].

[38] K. L. Smith, G. A. Shields, E. W. Bonning et al., A Search for Binary Active Galactic Nuclei: Double-peaked [O III] AGNs in the Sloan Digital Sky Survey, ApJ 716 (June, 2010) 866-877, [arXiv:0908.1998].

[39] X. Liu, J. E. Greene, Y. Shen et al., Discovery of Four kpc-scale Binary Active Galactic Nuclei, ApJ 715 (May, 2010) L30-L34, [arXiv: 1003.3467 ].

[40] Y. Shen, X. Liu, J. E. Greene et al., Type 2 Active Galactic Nuclei with Double-peaked [O III] Lines. II. Single AGNs with Complex Narrow-line Region Kinematics are More Common than Binary AGNs, ApJ 735 (July, 2011) 48-+, [arXiv: 1011.5246 ].

[41] D. J. Rosario, R. C. McGurk, C. E. Max et al., Adaptive Optics Imaging of Quasi-stellar Objects with Double-peaked Narrow Lines: Are They Dual Active Galactic Nuclei?, ApJ 739 (Sept., 2011) 44, [arXiv:1102.1733].

[42] J. M. Comerford, B. F. Gerke, D. Stern et al., Kiloparsec-scale Spatial Offsets in Double-peaked Narrow-line Active Galactic Nuclei. I. Markers for Selection of Compelling Dual Active Galactic Nucleus Candidates, ApJ 753 (July, 2012) 42, [arXiv:1111.2862].

[43] H. Fu, Z.-Y. Zhang, R. J. Assef et al., A Kiloparsec-scale Binary Active Galactic Nucleus Confirmed by the Expanded Very Large Array, ApJ 740 (Oct., 2011) L44, [arXiv:1109.0008].

[44] J. M. Comerford, D. Pooley, R. S. Barrows et al., Merger-driven Fueling of Active Galactic Nuclei: Six Dual and Offset AGNs Discovered with Chandra and Hubble Space Telescope Observations, ApJ 806 (June, 2015) 219, [arXiv: 1504.01391 ].

[45] F. Müller-Sánchez, J. M. Comerford, R. Nevin et al., The Origin of Double-peaked Narrow Lines in Active Galactic Nuclei. I. Very Large Array Detections of Dual AGNs and AGN Outflows, ApJ 813 (Nov., 2015) 103, [arXiv: 1509.04291$].$

[46] R. C. McGurk, C. E. Max, D. J. Rosario et al., Spatially Resolved Spectroscopy of SDSS J0952+2552: A Confirmed Dual Active Galactic Nucleus, ApJ 738 (Sept., 2011) L2, [arXiv:1107.2651]. 
[47] L. Blecha, A. Loeb, and R. Narayan, Double-peaked narrow-line signatures of dual supermassive black holes in galaxy merger simulations, MNRAS 429 (Mar., 2013) 2594-2616, [arXiv:1201.1904].

[48] V. Springel and L. Hernquist, Cosmological smoothed particle hydrodynamics simulations: a hybrid multiphase model for star formation, MNRAS 339 (Feb., 2003) 289-311, [astro-ph/0206393].

[49] V. Springel, The cosmological simulation code GADGET-2, MNRAS 364 (Dec., 2005) 1105-1134, [astro-ph/0505010].

[50] P. Jonsson, SUNRISE: polychromatic dust radiative transfer in arbitrary geometries, MNRAS 372 (Oct., 2006) 2-20, [astro-ph/ 0604118 ].

[51] P. Jonsson, B. A. Groves, and T. J. Cox, High-resolution panchromatic spectral models of galaxies including photoionization and dust, MNRAS 403 (Mar., 2010) 17-44.

[52] R. C. McGurk, C. E. Max, A. M. Medling et al., Spatially Resolved Imaging and Spectroscopy of Candidate Dual Active Galactic Nuclei, ApJ 811 (Sept., 2015) 14.

[53] C. Rodriguez, G. B. Taylor, R. T. Zavala et al., A Compact Supermassive Binary Black Hole System, ApJ 646 (July, 2006) 49-60, [astro-ph / 0604042 ].

[54] S. Burke-Spolaor, A radio Census of binary supermassive black holes, MNRAS 410 (Feb., 2011) 2113-2122, [arXiv: 1008 .4382].

[55] A. Escala, R. B. Larson, P. S. Coppi et al., The Role of Gas in the Merging of Massive Black Holes in Galactic Nuclei. II. Black Hole Merging in a Nuclear Gas Disk, ApJ 630 (Sept., 2005) 152-166, [astro-ph/0406304].

[56] J. Cuadra, P. J. Armitage, R. D. Alexander et al., Massive black hole binary mergers within subparsec scale gas discs, MNRAS 393 (Mar., 2009) 1423-1432, [arXiv : 0809.0311 ].

[57] M. C. Begelman, R. D. Blandford, and M. J. Rees, Massive black hole binaries in active galactic nuclei, Nature 287 (Sept., 1980) 307-309.

[58] Q. Yu, Evolution of massive binary black holes, MNRAS 331 (Apr., 2002) 935-958, [astro-ph/0109530].

[59] P. Berczik, D. Merritt, R. Spurzem et al., Efficient Merger of Binary Supermassive Black Holes in Nonaxisymmetric Galaxies, ApJ 642 (May, 2006) L21-L24, [astro-ph/0601698].

[60] F. M. Khan, A. Just, and D. Merritt, Efficient Merger of Binary Supermassive Black Holes in Merging Galaxies, ApJ 732 (May, 2011) 89, [arXiv:1103.0272].

[61] K. Holley-Bockelmann and F. M. Khan, Galaxy Rotation and Rapid Supermassive Binary Coalescence, ApJ 810 (Sept., 2015) 139, [arXiv: 1505.06203 ]. 
[62] M. J. Valtonen, H. J. Lehto, K. Nilsson et al., A massive binary black-hole system in OJ287 and a test of general relativity, Nature 452 (Apr., 2008) 851-853, [arXiv: 0809.1280 ].

[63] P. Tsalmantza, R. Decarli, M. Dotti et al., A Systematic Search for Massive Black Hole Binaries in the Sloan Digital Sky Survey Spectroscopic Sample, ApJ 738 (Sept., 2011) 20, [arXiv:1106.1180].

[64] M. Eracleous, T. A. Boroson, J. P. Halpern et al., A Large Systematic Search for Close Supermassive Binary and Rapidly Recoiling Black Holes, ApJS 201 (Aug., 2012) 23.

[65] Y. Shen, X. Liu, A. Loeb et al., Constraining Sub-parsec Binary Supermassive Black Holes in Quasars with Multi-epoch Spectroscopy. I. The General Quasar Population, ApJ 775 (Sept., 2013) 49, [arXiv:1306.4330].

[66] M. J. Graham, S. G. Djorgovski, D. Stern et al., A possible close supermassive black-hole binary in a quasar with optical periodicity, Nature 518 (Feb., 2015) 74-76, [arXiv:1501.01375].

[67] T. Liu, S. Gezari, S. Heinis et al., A Periodically Varying Luminous Quasar at $z=2$ from the Pan-STARRS1 Medium Deep Survey: A Candidate Supermassive Black Hole Binary in the Gravitational Wave-driven Regime, ApJ 803 (Apr., 2015) L16, [arXiv: 1503.02083 ].

[68] A. Sesana, Systematic investigation of the expected gravitational wave signal from supermassive black hole binaries in the pulsar timing band, MNRAS 433 (June, 2013) L1-L5, [arXiv:1211.5375].

[69] R. M. Shannon, V. Ravi, L. T. Lentati et al., Gravitational waves from binary supermassive black holes missing in pulsar observations, Science 349 (Sept., 2015) 1522-1525, [arXiv:1509.07320].

[70] A. Peres, Classical Radiation Recoil, Physical Review 128 (Dec., 1962) 2471-2475.

[71] J. D. Bekenstein, Gravitational-Radiation Recoil and Runaway Black Holes, ApJ 183 (July, 1973) 657-664.

[72] C. O. Lousto, Y. Zlochower, M. Dotti et al., Gravitational recoil from accretion-aligned black-hole binaries, Phys Rev D 85 (Apr., 2012) 084015, [arXiv: 1201.1923 ].

[73] T. Bogdanović, C. S. Reynolds, and M. C. Miller, Alignment of the Spins of Supermassive Black Holes Prior to Coalescence, ApJ 661 (June, 2007) L147-L150, [astro-ph/0703054].

[74] M. Dotti, M. Volonteri, A. Perego et al., Dual black holes in merger remnants - II. Spin evolution and gravitational recoil, MNRAS 402 (Feb., 2010) 682-690, [arXiv:0910.5729].

[75] M. C. Miller and J. H. Krolik, Alignment of Supermassive Black Hole Binary Orbits and Spins, ApJ 774 (Sept., 2013) 43, [arXiv:1307. 6569]. 
[76] L. Blecha, T. J. Cox, A. Loeb et al., Recoiling black holes in merging galaxies: relationship to active galactic nucleus lifetimes, starbursts and the $M_{B H^{-}} \sigma_{*}$ relation, MNRAS 412 (Apr., 2011) 2154-2182, [arXiv:1009.4940].

[77] D. Sijacki, V. Springel, and M. G. Haehnelt, Gravitational recoils of supermassive black holes in hydrodynamical simulations of gas-rich galaxies, MNRAS 414 (July, 2011) 3656-3670, [arXiv:1008.3313].

[78] J. Guedes, P. Madau, L. Mayer et al., Recoiling Massive Black Holes in Gas-rich Galaxy Mergers, ApJ 729 (Mar., 2011) 125, [arXiv: 1008.2032 ].

[79] L. Blecha and A. Loeb, Effects of gravitational-wave recoil on the dynamics and growth of supermassive black holes, MNRAS 390 (Nov., 2008) 1311-1325, [arXiv: 0805.1420 ].

[80] S. Komossa, H. Zhou, and H. Lu, A Recoiling Supermassive Black Hole in the Quasar SDSS J092712.65+294344.0?, ApJ 678 (May, 2008) L81-L84, [arXiv : 0804 . 4 585].

[81] A. Robinson, S. Young, D. J. Axon et al., Spectropolarimetric Evidence for a Kicked Supermassive Black Hole in the Quasar E1821+643, ApJ 717 (July, 2010) L122-L126, [arXiv:1006.0993].

[82] D. Batcheldor, A. Robinson, D. J. Axon et al., A Displaced Supermassive Black Hole in M87, ApJ 717 (July, 2010) L6-L10, [arXiv: 1005.2173 ].

[83] F. Civano, M. Elvis, G. Lanzuisi et al., A Runaway Black Hole in COSMOS: Gravitational Wave or Slingshot Recoil?, ApJ 717 (July, 2010) 209-222, [arXiv:10 03.0020 ].

[84] M. Koss, L. Blecha, R. Mushotzky et al., SDSS1133: an unusually persistent transient in a nearby dwarf galaxy, MNRAS 445 (Nov., 2014) 515-527, [arXiv: 1401.6798 ].

[85] J. M. Comerford, R. L. Griffith, B. F. Gerke et al., 1.75 h-1 kpc Separation Dual Active Galactic Nuclei at $z=0.36$ in the Cosmos Field, ApJ 702 (Sept., 2009) L82-L86.

[86] F. Civano, M. Elvis, G. Lanzuisi et al., Chandra High-resolution observations of CID-42, a Candidate Recoiling Supermassive Black Hole, ApJ 752 (June, 2012) 49, [arXiv:1205.0815].

[87] M. Novak, V. Smolčić, F. Civano et al., New insights from deep VLA data on the potentially recoiling black hole CID-42 in the COSMOS field, MNRAS 447 (Feb., 2015) 1282-1288, [arXiv:1412.0004].

[88] L. Blecha, F. Civano, M. Elvis et al., Constraints on the nature of CID-42: recoil kick or supermassive black hole pair?, MNRAS 428 (Jan., 2013) 1341-1350, [arXiv:1205.6202].

[89] E. W. Bonning, G. A. Shields, and S. Salviander, Recoiling Black Holes in Quasars, ApJ 666 (Sept., 2007) L13-L16, [arXiv: 0705 . 4263]. 
[90] D. Lena, A. Robinson, A. Marconi et al., Recoiling Supermassive Black Holes: A Search in the Nearby Universe, ApJ 795 (Nov., 2014) 146, [arXiv:1409.3976].

[91] L. Blecha, D. Sijacki, L. Z. Kelley et al., Recoiling black holes: prospects for detection and implications of spin alignment, MNRAS 456 (Feb., 2016) 961-989, [arXiv:1508.01524].

[92] M. Volonteri and P. Madau, Off-Nuclear AGNs as a Signature of Recoiling Massive Black Holes, ApJ 687 (Nov., 2008) L57-L60, [arXiv: 0809.4007 ].

[93] M. Vogelsberger, S. Genel, V. Springel et al., Properties of galaxies reproduced by a hydrodynamic simulation, Nature 509 (May, 2014) 177-182, [arXiv: 1405.1418 ].

[94] M. Vogelsberger, S. Genel, V. Springel et al., Introducing the Illustris Project: simulating the coevolution of dark and visible matter in the Universe, MNRAS 444 (Oct., 2014) 1518-1547, [arXiv:1405.2921].

[95] S. Genel, M. Vogelsberger, V. Springel et al., Introducing the Illustris project: the evolution of galaxy populations across cosmic time, MNRAS 445 (Nov., 2014) 175-200, [arXiv:1405.3749].

[96] D. Nelson, A. Pillepich, S. Genel et al., The illustris simulation: Public data release, Astronomy and Computing 13 (Nov., 2015) 12-37, [arXiv: 1504.00362 ].

[97] V. Springel, E pur si muove: Galilean-invariant cosmological hydrodynamical simulations on a moving mesh, MNRAS 401 (Jan., 2010) 791-851, [arXiv: 0901.4107 ].

[98] L. C. Ho, A. V. Filippenko, and W. L. W. Sargent, The Influence of Bars on Nuclear Activity, ApJ 487 (Oct., 1997) 591-602, [astro-ph/ 9704100 ].

[99] M. A. Galloway, K. W. Willett, L. F. Fortson et al., Galaxy Zoo: the effect of bar-driven fuelling on the presence of an active galactic nucleus in disc galaxies, MNRAS 448 (Apr., 2015) 3442-3454, [arXiv: 1502.01033].

[100] I. Shlosman, J. Frank, and M. C. Begelman, Bars within bars - A mechanism for fuelling active galactic nuclei, Nature 338 (Mar., 1989) 45-47.

[101] P. F. Hopkins and E. Quataert, How do massive black holes get their gas?, MNRAS 407 (Sept., 2010) 1529-1564, [arXiv:0912.3257].

[102] J. Kormendy and L. C. Ho, Coevolution (Or Not) of Supermassive Black Holes and Host Galaxies, ARA\&A 51 (Aug., 2013) 511-653, [arXiv:1304 . 7762].

[103] D. Sijacki, M. Vogelsberger, S. Genel et al., The Illustris simulation: the evolving population of black holes across cosmic time, MNRAS 452 (Sept., 2015) 575-596, [arXiv:1408.6842]. 